\title{
Time and number sense develop in tandem?
}

\author{
Mark J. Yates* \\ Department of Psychology, University of Melbourne, Parkville, VIC, Australia \\ *Correspondence: mjyates@unimelb.edu.au
}

\section{A commentary on}

Developmental neuroscience of time and number: implications for autism and other neurodevelopmental disabilities by Allman, M. J., Pelphrey, K. A., and Meck, W. H. (2012). Front. Integr. Neurosci. 6:7 doi: 10.3389/fnint.2012.00007

The last two decades have seen a marked rise in interest in what might be termed "time sense" and "number sense" and the potential relationship between them (for reviews see Buhusi and Meck, 2005; Bueti and Walsh, 2009; Nieder and Dehaene, 2009). "Time sense" refers to the ability that allows human adults (as well as infants and animals) to discriminate which of two sequentially presented stimuli is greater in duration. "Number sense" refers to the ability to determine - in the absence of explicit counting - which of two stimuli (each comprising of a number of separate elements) contains the greater number of elements. The "elements" in question might be presented simultaneously (e.g., visual dots in an array) or sequentially (e.g., a series of taps to the arm).

A major new review appearing within this issue (Allman et al., 2012) provides an update on research into both time and number sense. Unique to this review is its focus on the developmental trajectory of time and number sense. This is paired with an overview of developmental disorders in which basic time and/or number sense appears to be impaired, and coverage of findings concerning the neural correlates of time and number representation.

Charting the developmental trajectory of time and number sense potentially provides insights beyond those that can be gleaned from a "snapshot" of the same abilities in their mature form in adulthood. One obvious advantage is that characterizing time and number sense at certain critical moments of development (e.g., at birth) can differentiate, for instance, between innate versus experience-dependent aspects of time and number abilities, or between aspects of time and number sense that are present prior to exposure with cultural number symbol systems versus those that emerge after such exposure.

A second advantage is the opportunity it provides to shed light on the question of whether a single "magnitude accumulation" system underlies both time and number perception (and possibly other dimensions as well), as proposed by two theoretical models discussed in the review (Meck and Church, 1983; Walsh, 2003). Over the course of normal development, performance on both numerosity and duration discrimination tasks improves with signature trajectories. In theory, if these changes in acuity occur in lockstep across the two different dimensions, this would lend at least prima facie support for the existence of a common magnitude processing mechanism that is recruited during both types of discrimination task. Such evidence would be more convincing than mere similarities in performance for time and number discrimination tasks at any given snapshot of time. In practice, the picture is more complex. Various lines of evidence indicate that time sense and number sense do in fact appear to develop at least roughly in tandem, but there are many caveats: discrimination performance sometimes improves for a selected segment of the range of possible durations/ numerosities rather than uniformly across the entire range, numerosity discrimination for sequences of elements differs from that for simultaneously presented elements, improvements in discrimination performance over development are not unique to time and number sense but rather reflect a general feature of perceptual abilities, and so on. Furthermore, even if it could be shown that the developmental trajectories of time and number sense are perfectly synchronized, this could not be counted as definitive support for shared time and number magnitude processing given that simultaneous improvements in discrimination performance across the two dimensions might also be explained by the development/improvement of a third factor (such as attention or working memory) which contributes to both types of discrimination task. Nevertheless, despite the complexities, a systematic investigation of how time and number sense become refined over the course of development may yet prove to be very informative for the shared magnitude representation hypothesis, currently an especially active area of interest within cognitive neuroscience.

Finally, a sharper picture of the trajectory of time and number sense during typical development provides an important baseline against which atypical development of these abilities can be better delineated and understood. In the latter part of the review, the focus turns to developmental disorders which possibly stem from, or are at least in some way associated with, impairments in basic time and number sense. The most obvious example is developmental dyscalculia, a disorder marked by selective impairment of numerical/mathematical ability against a background of otherwise normal academic achievement. While there is still considerable debate about the nature of the underlying deficit in this disorder, there is evidence that it involves an impairment of basic number sense (i.e., non-symbolic numerical processing; e.g., Price et al., 2007) though an alternative account is that the deficit is in linking number symbols with non-symbolic numerical magnitudes (i.e., attaching meaning to numerical symbols; e.g., Rousselle and Noël, 2007). Interestingly, the study of developmental dyscalculia can also assist in clarifying the relationship between time and number sense. If basic number sense is impaired in dyscalculia, and if time and number sense are subserved by a fully shared magnitude system, then time sense should also be impaired in this disorder. Cappelletti et al. (2011) recently tested this prediction, and found that basic time sense is not impaired in 
developmental dyscalculia (at least not in the sub-second duration range). However, they found that numbers did interfere with temporal processing, both in those with dyscalculia and controls, though in different ways. They concluded that their data supported the existence of a partially shared magnitude system for number and time, as this is the only possibility that can account for both dissociations as well as interactions between number and time sense, as were observed.

There is now also increasing interest in the possibility that ADHD and autism spectrum disorder involve an impairment of basic time sense and/or number sense (e.g., Boucher, 2001; Allman, 2011; Gooch et al., 2011). An intriguing hypothesis put forward by the review authors is that the striking preoccupation with symbolic numbers commonly observed in autism spectrum disorder - along with unusual levels of interest in timetables, calendars, and routines (these latter three can be conceived as "external" temporal supports - Lalli et al., 1994) - may, in fact, reflect an adaptive strategy to compensate for a deficit in basic time sense. Such speculations are to be encouraged. Arguably somewhat belatedly, time and number sense are now recognized as fundamental mental abilities integral to perception and behavior. An increased focus on the possible clinical manifestations of disordered time and number sense can only be welcomed.

\section{REFERENCES}

Allman, M. J.(2011). Deficits in temporal processing associated with autistic disorder. Front. Integr. Neurosci. 5:2. doi: 10.3389/fnint.2011.00002

Allman, M. J., Pelphrey, K. A., and Meck, W. H. (2012). Developmental neuroscience of time and number: implications for autism and other neurodevelopmental disabilities. Front. Integr. Neurosci. 6:7. doi: 10.3389/ fnint.2012.00007

Boucher, J. (2001). "Lost in a sea of time: time-parsing and autism," in Time and Memory, eds C. Hoerl and T. McCormack (Oxford: Oxford University Press), 111-135.

Bueti, D., and Walsh, V. (2009). The parietal cortex and the representation of time, space, number and other magnitudes. Philos. Trans. R. Soc. Lond. B Biol. Sci. 364, 1831-1840.

Buhusi, C. V., and Meck, W. H. (2005). What makes us tick? Functional and neural mechanisms of interval timing. Nat. Rev. Neurosci. 6, 755-765.

Cappelletti, M., Freeman, E. D., and Butterworth, B. L. (2011). Time processing in dyscalculia. Front. Psychol. 2:364. doi: 10.3389/fpsyg.2011.00364

Gooch, D., Snowling, M., and Hulme, C. (2011). Time perception, phonological skills and executive function in children with dyslexia and/or ADHD symptoms. J. Child Psychol. Psychiatry 52, 195-203.

Lalli, J. S., Casey, S., Goh, H., and Merlino, J. (1994). Treatment of escape-maintained aberrant behaviour with escape extinction and predictable routines. J. Appl. Behav. Anal. 27, 705-714.

Meck, W. H., and Church, R. M. (1983). A mode control model of counting and timing processes. J. Exp. Psychol. Anim. Behav. Process. 9, 320-334.

Nieder, A., and Dehaene, S. (2009). Representation of number in the brain. Annu. Rev. Neurosci.32, 185-208.

Price, G. R., Holloway, I., Räsänen, P., Vesterinen, M., and Ansari, D. (2007). Impaired parietal magnitude processing in developmental dyscalculia. Curr. Biol. 17, R1042-R1043.

Rousselle, L., and Noël, M.P.(2007). Basic numerical skills in children with mathematics learning disabilities: a comparison of symbolic vs. non-symbolic number magnitude processing. Cognition 102, 361-395.

Walsh, V. (2003). A theory of magnitude: common cortical metrics of time, space and quantity. Trends Cogn. Sci. (Regul. Ed.) 7, 483-488.

Received: 23 February 2012; accepted: 29 February 2012; published online: 16 March 2012.

Citation: Yates MJ (2012) Time and number sense develop in tandem? Front. Integr. Neurosci. 6:12. doi: 10.3389/ fnint.2012.00012

Copyright $(92012$ Yates. This is an open-access article distributed under the terms of the Creative Commons Attribution Non Commercial License, which permits non-commercial use, distribution, and reproduction in other forums, provided the original authors and source are credited. 